\title{
Prognostic Impact of Elevation of Vascular Endothelial Growth Factor Family Expression in Patients with Non-small Cell lung Cancer: an Updated Meta-analysis
}

\author{
Chun-Long Zheng ${ }^{1 \&}$, Chen Qiu ${ }^{2 \&}$, Mei-Xiao Shen², Xiao Qu², Tie-Hong Zhang ${ }^{2}$, \\ Ji-Hong Zhang' ${ }^{2}$ Jia-Jun Du ${ }^{1,2 *}$
}

\begin{abstract}
Background: The vascular endothelial growth factor family has been implicated in tumorigenesis and metastasis. The prognostic value of each vascular endothelial growth factor family member, particular VEGF/ VEGFR co-expression, in patients with non-small lung cancer remains controversial. Materials and Methods: Relevant literature was identified by searching PubMed, EMBASE and Web of Science. Studies evaluating expression of VEGFs and/or VEGFRs by immunohistochemistry or ELISA in lung cancer tissue were eligible for inclusion. Hazard ratios (HRs) and 95\% confidence intervals (CIs) from individual study were pooled by using a fixed- or random-effect model, heterogeneity and publication bias analyses were also performed. Results: 74 studies covering 7,631 patients were included in the meta-analysis. Regarding pro-angiogenesis factors, the expression of VEGFA (HR=1.633, 95\% CI: 1.490-1.791) and VEGFR1 (HR=1.924, 95\% CI: 1.220-3.034) was associated separately with poor survival. Especially, VEGFA over-expression was an independent prognostic factor in adenocarcinoma (ADC) (HR=1.775,95\% CI: 1.384-2.275) and SCC (HR=2.919, 95\% CI: 2.060-4.137). Co-expression of VEGFA/VEGFR2 (HR=2.011,95\% CI: 1.405-2.876) was also significantly associated with worse survival. For lymphangiogenesis factors, the expression of VEGFC (HR=1.611, 95\% CI: 1.407-1.844) predicted a poor prognosis. Co-expression of VEGFC/VEGFR3 (HR=2.436, 95\% CI: 1.468-4.043) emerged as a preferable prognostic marker. Conclusions: The expression of VEGFA (particularly in SCC and early stage NSCLC), VEGFC, VEGFR1 indicates separately an unfavorable prognosis in patients with NSCLC.Co-expression VEGFA/ VEGFR2 is comparable with VEGFC/VEGFR3, both featuring sufficient discrimination value as preferable as prognostic biologic markers.
\end{abstract}

Keywords: Vascular endothelial growth factor - vascular endothelial growth factor receptor - prognosis - NSCLC

Asian Pac J Cancer Prev, 16 (5), 1881-1895

\section{Introduction}

Lung cancer is the leading cause of cancer-related mortality around the world (Alberg and Samet, 2003). Less than $15 \%$ of the patients will be cured and enjoy long-term survival. The poor prognosis has shown little improvement in recent decades (Molina et al., 2008). In light of disappointing therapeutic effect it is likely that the use of clinical or molecular markers will become important in predicting response to treatment and outcome. The main prognostic factors in NSCLC are disease stage, amount of weight lost, microvessel density, ERCC1, RRM1, BRCA1, p53, bcl-2, KRAS, Ki-67, (18)F-FDG, AKT, mTOR and EGFR mutation (Paesmans et al., 1995; Kaira and Yamamoto, 2010). However, these biological prognostic factors didn't well predict clinical outcome or their discriminate value is insufficient to predict the optimal therapeutic course for an individual. Therefore, it is important to identify ideal predictive/prognostic biologic markers for patients undergoing treatment.

The hypothesis "Tumor growth is angiogenesis dependent" was first proposed by Folkman in 1971 (Folkman, 1971), which was confirmed by subsequent observations that tumors are strictly limited in size in the absence of neovascularization (Gimbrone et al., 1972). Angiogenesis, the formation of blood vessels from preexisting vessels at a later stage (Ferrara and Kerbel, 2005), is critical for the development and subsequent growth of tumors and is a prerequisite for metastasis. The VEGF family comprises four ligands (VEGFA, VEGFB, VEGFC and VEGFD), which exhibit specific binding profiles with three transmembrane VEGF receptors (VEGFR1, 2 and 3) and promote intracellular tyrosine kinase cascades when activated (Ferrara, 2002; Hicklin and Ellis, 2005). VEGFA and its receptor (VEGFR1, VEGFR2) play a major role in physiological as well as pathological angiogenesis 
including tumor angiogenesis. While VEGFC/D and their receptor VEGFR3 can regulate angiogenesis at early embryogenesis but mostly function as critical regulators of lymphangiogenesis during lifetime (Alitalo and Carmeliet, 2002; He et al., 2005).

The effect of VEGFs and/or VEGFRs expression on survival in patients with NSCLC has been studied for over decades. However, conflicting results regarding the ability of VEGF to predict survival have been reported one by one from different laboratories. In this study, we sought to conduct a systematic review with meta-analysis to primarily estimate the prognostic impact of each VEGF family member for survival in NSCLC patients. The secondary goal of our study was to explore whether the VEGF/VEGFR co-expression with sufficient discriminate value as preferable predictive/prognostic biologic marker for an individual undergoing treatment.

\section{Materials and Methods}

\section{Literature search}

An electronic literature search was performed to identify potentially relevant published article in Pub Med, EMBASE, Web of Science. We used lung cancer/ lung tumor/lung neoplasm/lung carcinoma and vascular endothelial growth factor/VEGF and prognosis/prognostic as searching terms. No lower date limit was used and last search was updated on May 9, 2014. All potentially eligible studies were retrieved. The study protocol conforms to the ethical guidelines of the Declaration of Helsinki, which was approved by the ethics committee of Provincial Hospital Affiliated to Shandong University.

\section{Inclusion and study criteria}

To be eligible studies for inclusion in this metaanalysis had to meet the following inclusion criteria: (i) measure VEGF and/or VEGFRs expression in the primary lung cancer tissue with immunohistochemistry (IHC) or enzyme linked immune sorbent assay (ELISA); (ii) the study has sufficient data on survival for extracting; (iii) the same patient series was included in more than one publication, only the more recent or most complete study was included in the analysis.

In addition, the expression of different VEGFs and/or VEGFRs obtained from the same patient population by the same authors in different years was also included; There was no pre-specified sample size or follow-up period used to determine study inclusion. Criteria used to determine duplicate populations included study period, treatment information, and any additional inclusion criteria. Language was restricted for review title and abstract in English, but was not restricted for data collection.

\section{Data extraction}

Data were extracted using a predefined form, recording: first author, year of publication, number of patients, histology, disease stage, detective method, cutoffs and positive ratios of positive expression, outcome of univariate or multivariate analysis (HRs, 95\% CIs) and the original author's results (Table 1). If the above data of any categories was not reported in the primary study, items were treated as "NR" (not report). We did not contact the author of the primary study to request the information. The required information was extracted independently from primary studies by two reviewers (Chunlong Zheng and Chen Qiu) according to a standard data record worksheet designed in advance.

\section{Statistical}

All calculations were performed with the hazard ratios (HRs) and the associated 95\% confidence intervals (CIs) of OS, RFS, DSS, or DFS. The most accurate and easiest method was to collect the reported HRs and CIs from primary articles directly. When these statistical variables were not given explicitly in an article, which were either extracted from the Kaplan-Meier survival curves indirectly or calculated, if available number (the total numbers of events and the total numbers of patients in each group) were given, assuming that the rate of censored patients was constant during the study follow-up. The method and spreadsheet used for these calculations were provided by Tierney et al (Tierney et al., 2007). HRs defined as the risk of death or progression for high expression vs low expression. In studies that reported HRs for low level vs high level, the reciprocal of the HR calculated and $p$ value were taken to calculate the associated $95 \%$ CI for meta-analysis.

Heterogeneity of the individual HRs was performed with Q statistic test and $\mathrm{I}^{2}$ statistic test. All of the studies included were categorized by VEGF and VEGFRs isoform, histology, disease stage, patient race. Individual meta-analysis was conducted in each subgroup. If HRs were found to have fine homogeneity $(p(\mathrm{Q})>0.05$, $\mathrm{I}^{2}<56 \%$ ) (Walter, 1997; Hardy and Thompson, 1998; Dwyer et al., 2001; Higgins and Thompson, 2002), a fixed effect model was used for secondary analysis; if not, a random-effect model was used. In this meta-analysis, Inverse Variance fixed effects and I-V heterogeneity random effects analysis were used to estimate the effect of VEGF family high expression on survival. By convention, an observed $\mathrm{HR}>1$ implies worse survival with positive expression, and the impact on survival was considered to be statistically significant if the $95 \% \mathrm{CI}$ didn't overlap with 1 . Similarly, if $\mathrm{HR}<1$ means good survival, and statistically significant if the $95 \%$ CI didn't overlap with 1 . Horizontal lines represent $95 \%$ CIs. Each square represents the HR point estimate, and its area is proportional to the weight of the study. The diamond (and broken line) represents the overall summary estimate, with CI represented by its width. The unbroken vertical line is set at the null value $(\mathrm{HR}=1.0)$.

Evidence of publication bias was sought using the methods of Egger et al. (1997) and Begg et al. (1994). Moreover, contour-enhanced funnel plot (Peters et al., 2008) was performed constructed to assess publication and/or selection bias. If studies appear to be missing in areas of low statistical significance, then it is possible that the asymmetry is due to publication bias. If studies seem to be missing in areas of high statistical significance, then publication bias is a less likely cause of the funnel asymmetry. Intercept significance was determined by the test suggested by Begg and Egger $(p<0.05$ was considered 
DOI:http://dx.doi.org/10.7314/APJCP.2015.16.5.1881

VEGF Family Expression and Prognosis of Non-small Cell Lung Cancer

Table 1. Characteristics and Results of the Eligible Studies

\begin{tabular}{|c|c|c|c|c|c|c|c|c|c|c|c|}
\hline $\begin{array}{l}\text { First } \\
\text { Author }\end{array}$ & Year & $\begin{array}{l}\text { Patients } \\
\text { Source }\end{array}$ & $\begin{array}{l}\text { No. } \\
\text { of } \\
\text { Pts }\end{array}$ & $\begin{array}{l}\text { Histol- } \\
\text { ogy }\end{array}$ & Stage & $\begin{array}{l}\text { Meth- } \\
\text { od }\end{array}$ & $\begin{array}{l}\text { Cut- } \\
\text { off } \\
(\%)\end{array}$ & Positive $(\%)$ & $\begin{array}{c}\text { HR } \\
\text { Estimation }\end{array}$ & $\mathrm{HR}(95 \% \mathrm{CI})$ & $\begin{array}{l}\text { Re- } \\
\text { sults }\end{array}$ \\
\hline Kim & 2013 & $\begin{array}{l}\text { south } \\
\text { korea }\end{array}$ & 75 & NSCLC & I-IIB & IHC & 20 & $\begin{array}{l}\text { VEGFA } \\
28 \%\end{array}$ & Report & $\begin{array}{c}0.86 \\
(0.111-6 . .658)\end{array}$ & $+\mathrm{ve}$ \\
\hline Yurdakul & 2012 & Turkey & 91 & NSCLC & I-IV & IHC & $\mathrm{CS}$ & $\begin{array}{l}\text { VEGFA } \\
37.4 \%\end{array}$ & Surv.curves & $\begin{array}{c}1.25 \\
(0.80-1.96)\end{array}$ & -ve \\
\hline Wang & 2012 & China & 210 & NSCLC & III & IHC & $\mathrm{CS}$ & $\begin{array}{l}\text { VEGFA } \\
49.0 \%\end{array}$ & Report & $\begin{array}{c}1.461 \\
(1.015-2.104)\end{array}$ & $+\mathrm{ve}$ \\
\hline Starnes & 2012 & USA & 102 & NSCLC & I & IHC & 50 & $\begin{array}{l}\text { KDR } \\
49 \%\end{array}$ & Report & $\begin{array}{c}1.258 \\
(0.510-3.102)\end{array}$ & $-\mathrm{ve}$ \\
\hline $\begin{array}{l}\text { Anagnos- } \\
\text { tou }\end{array}$ & 2011 & Greece & $\begin{array}{l}97 \\
59 \\
47\end{array}$ & $\begin{array}{l}\text { NSCLC } \\
\text { NSCLC } \\
\text { ADC }\end{array}$ & $\begin{array}{l}\text { I-IV } \\
\text { I-III }\end{array}$ & IHC & $\mathrm{CS}$ & $\begin{array}{c}\text { VEGFA } \\
66.0 \% \\
\text { VEGFC } \\
80.6 \% \\
\text { VEGFD } \\
92.2 \% \\
\text { VEGFR3 } \\
94.2 \%\end{array}$ & Report & $\begin{array}{c}\text { A 1.4 } \\
(0.25-7.9) \\
\text { C } 4 \\
(1.11-14.27) \\
\text { D } 0.61 \\
(0.26-1.42) \\
\text { R3 0.85 } \\
(0.08-8.46) \\
\text { A } 0.11 \\
(0.002-5.2) \\
\text { C } 10.6 \\
(4.3-26.5) \\
\text { D } 0.75 \\
(0.23-2.4) \\
\text { R3 0.01 } \\
(0-0.34) \\
\text { A } 8.6 \\
(1.4-53.6)\end{array}$ & $\begin{array}{l}\text {-ve } \\
+\mathrm{ve} \\
\text {-ve } \\
\text {-ve } \\
\text {-ve } \\
+\mathrm{ve} \\
\text {-ve } \\
\text { In- } \\
\text { verse } \\
+\mathrm{ve}\end{array}$ \\
\hline Ucvet & 2011 & Turkey & 117 & NSCLC & $\begin{array}{l}\text { IA- } \\
\text { IIIB }\end{array}$ & IHC & 25 & $\begin{array}{l}\text { VEGFA } \\
70.1 \%\end{array}$ & Surv.curves & $\begin{array}{c}0.92 \\
(0.50-1.69) \\
\end{array}$ & $-\mathrm{ve}$ \\
\hline Donnem & 2011 & Norway & 335 & NSCLC & I-IIIA & IHC & CS & $\begin{array}{c}A+\mathrm{R} 2 \\
29 \% \\
\end{array}$ & Surv.curves & $\begin{array}{l}A+R 21.85 \\
(1.16-2.96) \\
\end{array}$ & $+\mathrm{ve}$ \\
\hline Dai & 2011 & China & 98 & NSCLC & I-IIIa & $\mathrm{IHC}$ & CS & $\begin{array}{l}\text { VEGFC } \\
59.2 \%\end{array}$ & Report & $\begin{array}{c}1.862 \\
(1.464-2.386)\end{array}$ & $+\mathrm{ve}$ \\
\hline Chen & 2011 & Taiwan & 49 & NSCLC & I & IHC & 10 & VEGFC $49 \%$ & Report & $\begin{array}{c}3.98 \\
(1.23-12.89) \\
\end{array}$ & $+\mathrm{ve}$ \\
\hline Yamashita & 2010 & Japan & 117 & NSCLC & I & IHC & $\begin{array}{l}\text { A } 5 \\
\text { C } 10\end{array}$ & $\begin{array}{c}\text { VEGFA } \\
73.5 \% \\
\text { VEGFC } \\
48.7 \%\end{array}$ & Report & $\begin{array}{c}1.529 \\
(0.711-3.289) \\
1.359 \\
(0.708-2.604)\end{array}$ & $\begin{array}{l}-v e \\
-v e\end{array}$ \\
\hline Rades & 2010 & $\begin{array}{l}\text { Ger- } \\
\text { many }\end{array}$ & 61 & NSCLC & II-III & IHC & 10 & $\begin{array}{l}\text { VEGFA } \\
65.6 \% \\
\end{array}$ & Surv.curves & $\begin{array}{c}0.61 \\
(0.26-1.46) \\
\end{array}$ & -ve \\
\hline Lin & 2010 & China & 185 & NSCLC & I & IHC & 30 & $\begin{array}{l}\text { VEGFA } \\
49.7 \%\end{array}$ & Report & $\begin{array}{c}1.83 \\
(1.25-2.69)\end{array}$ & $+\mathrm{ve}$ \\
\hline Chen & 2010 & China & 92 & NSCLC & IIIA & $\mathrm{IHC}$ & 30 & $\begin{array}{c}\text { VEGFC } \\
65 \% \\
\end{array}$ & Report & $\begin{array}{c}2.523 \\
(1.057-6.024) \\
\end{array}$ & $+\mathrm{ve}$ \\
\hline Bircan & 2010 & Turkey & 46 & NSCLC & I-IV & IHC & NR & $\begin{array}{l}\text { VEGFA } \\
93.5 \%\end{array}$ & Report & $\begin{array}{c}2.452 \\
(0.457-13.166) \\
\end{array}$ & -ve \\
\hline Sun & 2009 & China & 82 & NSCLC & I-IIIA & IHC & 10 & $\begin{array}{l}\text { VEGFC } \\
74.4 \%\end{array}$ & DC & $\begin{array}{c}1.441 \\
(0.511-4.066) \\
\end{array}$ & -ve \\
\hline Carrillo & 2009 & Spain & 48 & NSCLC & I-IV & IHC & $\mathrm{CS}$ & $\begin{array}{c}\text { VEGFD } \\
22.9 \% \\
\text { VEGFR1 } \\
41.7 \% \\
\end{array}$ & Report & $\begin{array}{c}0.22 \\
(0.08-0.67) \\
3.56 \\
(1.56-8.1) \\
\end{array}$ & $\begin{array}{l}\text { In- } \\
\text { verse } \\
\text { +ve }\end{array}$ \\
\hline Bonnesen & 2009 & $\begin{array}{l}\text { Den- } \\
\text { mark }\end{array}$ & 102 & NSCLC & I-IIIB & IHC & CS & $\begin{array}{c}\text { VEGFA } \\
96.1 \% \\
\text { VEGFR2 } \\
93.1 \%\end{array}$ & Surv.curves & $\begin{array}{c}0.85 \\
(0.48-1.50) \\
0.73 \\
(0.45-1.16)\end{array}$ & $\begin{array}{l}-\mathrm{ve} \\
-\mathrm{ve}\end{array}$ \\
\hline
\end{tabular}


Chun-Long Zheng et al

Table 1 (cont). Characteristics and Results of the Eligible Studies

\begin{tabular}{|c|c|c|c|c|c|c|c|c|c|c|c|}
\hline Ko & 2008 & Korea & 118 & NSCLC & I-III & IHC & CS & $\begin{array}{c}\text { VEGFC } \\
60.2 \% \\
\text { VEGFD } \\
52.5 \%\end{array}$ & Surv.curves & $\begin{array}{c}0.65 \\
(0.31-1.35) \\
0.95 \\
(0.49-1.81)\end{array}$ & $\begin{array}{l}-v e \\
-v e\end{array}$ \\
\hline Kadota & 2008 & Japan & 147 & NSCLC & I-III & IHC & 0.3 & $\begin{array}{c}\text { VEGFA } \\
46.9 \% \\
\text { VEGFC } \\
44.2 \%\end{array}$ & Report & $\begin{array}{c}2.006 \\
(1.144-3.519) \\
1.801 \\
(1.024-3.167)\end{array}$ & $\begin{array}{l}+\mathrm{ve} \\
+\mathrm{ve}\end{array}$ \\
\hline Zhou & 2007 & China & 118 & NSCLC & I & IHC & 25 & $\begin{array}{l}\text { VEGFA } \\
36.5 \%\end{array}$ & $\mathrm{DE}$ & $\begin{array}{c}2.960 \\
(1.551-5.650) \\
\end{array}$ & $+\mathrm{ve}$ \\
\hline Yoo & 2007 & Korea & 219 & NSCLC & I-III & IHC & 5 & $\begin{array}{l}\text { VEGFA } \\
92.7 \%\end{array}$ & Report & $\begin{array}{c}1.063 \\
(0.365-3.096) \\
\end{array}$ & -ve \\
\hline Yilmaz & 2007 & Turkey & 50 & NSCLC & I-IIIA & IHC & 50 & VEGFA $26 \%$ & Report & $\begin{array}{c}4.651 \\
(1.538-14.069) \\
\end{array}$ & +ve \\
\hline Saintigny & 2007 & France & 92 & NSCLC & I-III & IHC & CS & $\begin{array}{c}\text { VEGFC 74\% } \\
\text { VEGFR3 } \\
42 \% \\
\text { C+R3 } \\
43.5 \%\end{array}$ & Report & $\begin{array}{c}1.237 \\
(0.644-2.376) \\
1.759 \\
(0.876-2.512) \\
1.819 \\
(0.925-3.578) \\
\end{array}$ & $\begin{array}{l}\text {-ve } \\
\text {-ve } \\
\text {-ve }\end{array}$ \\
\hline Ohta & 2007 & Japan & 122 & NSCLC & I & IHC & $\begin{array}{l}\text { A } 50 \\
\text { C } 10\end{array}$ & $\begin{array}{c}\text { VEGFA } \\
80.3 \% \\
A+C \\
23 \%\end{array}$ & Report & $\begin{array}{c}1.503 \\
(1.048-2.117) \\
1.365 \\
(0.964-1.966)\end{array}$ & $\begin{array}{l}+\mathrm{ve} \\
+\mathrm{ve}^{*}\end{array}$ \\
\hline Donnem & 2007 & Norway & 335 & NSCLC & I-IIIa & IHC & CS & $\begin{array}{c}\text { VEGFA:42\% } \\
\text { VEGFC:31\% } \\
\text { VE-FD:68\% } \\
\text { VEGFR1: } \\
25.7 \% \\
\text { VEGFR2: } \\
58 \% \\
\text { VEGFR3: } \\
57 \%\end{array}$ & Surv.curves & $\begin{array}{c}\text { A } 1.83 \\
(1.33-2.54) \\
\text { C } 1.43 \\
(0.89-2.34) \\
\text { D } 1.08 \\
(0.58-2.01) \\
\text { R1: } 1.66 \\
(1.2-2.3) \\
\text { R2: } 1.50 \\
(1.07-2.09) \\
\text { R3: } 1.686 \\
(1.153-2.446) \\
\end{array}$ & $\begin{array}{l}+\mathrm{ve} \\
-\mathrm{ve} \\
-\mathrm{ve} \\
+\mathrm{ve} \\
+\mathrm{ve} \\
+\mathrm{ve}\end{array}$ \\
\hline Maekawa & 2007 & Japan & 55 & $\mathrm{ADC}$ & I-III & $\begin{array}{l}\text { ELI- } \\
\text { SA }\end{array}$ & $\begin{array}{l}C \\
93.75 \\
D \\
62.50 \\
R 3\end{array}$ & NR & $\begin{array}{l}\text { Report } \\
\text { DC } \\
\text { Report }\end{array}$ & $\begin{array}{c}2.306 \\
(0.530-10.038) \\
0.294 \\
(0.096-0.896) \\
0.371 \\
(0.049-2.793)\end{array}$ & $\begin{array}{l}\text {-ve } \\
\text { In- }\end{array}$ \\
\hline Zhang & 2006 & China & 42 & NSCLC & I-IV & IHC & 10 & $\begin{array}{l}\text { VEGFC } \\
54.8 \%\end{array}$ & Surv.curves & $\begin{array}{c}3.07 \\
(1.22-7.69) \\
\end{array}$ & $+\mathrm{ve}$ \\
\hline Seto & 2006 & Japan & 60 & NSCLC & I & IHC & 10 & $\begin{array}{c}\text { VEGFA } 30 \% \\
\text { flt-1 32\% } \\
\text { KDR } 48 \% \\
\text { A+KDR } 23 \%\end{array}$ & $\begin{array}{l}\text { Surv.curves } \\
\text { Surv.curves } \\
\text { Surv.curves } \\
\text { DE }\end{array}$ & $\begin{array}{c}3.29 \\
(1.23-8.82) \\
1.96 \\
(0.88-4.37) \\
2.24 \\
(1.00-5.02) \\
2.26 \\
(1.297-3.938)\end{array}$ & $\begin{array}{l}\text { +ve } \\
\text {-ve } \\
+\mathrm{ve} \\
+\mathrm{ve}\end{array}$ \\
\hline Enatsu & 2006 & Japan & 78 & $\mathrm{ADC}$ & I-III & IHC & 10 & $\begin{array}{l}\text { VEGFA: } 68 \% \\
\text { VEGFC } 42 \%\end{array}$ & Report & $\begin{array}{c}0.35 \\
(0.093-1.332) \\
0.471 \\
(0.141-1.574) \\
\end{array}$ & $\begin{array}{l}-\mathrm{ve} \\
-\mathrm{ve}\end{array}$ \\
\hline Tomita & 2005 & Japan & 60 & NSCLC & I-III & IHC & 20 & VEGFA 58\% & Report & $\begin{array}{c}0.950 \\
(0.729-2.484) \\
\end{array}$ & -ve \\
\hline Renyi & 2005 & $\begin{array}{l}\text { Hun- } \\
\text { gary }\end{array}$ & 103 & NSCLC & I-IIIA & IHC & 30 & $\begin{array}{l}\text { VEGFC } \\
54.4 \%\end{array}$ & DC & $\begin{array}{c}1.756 \\
(0.627-2.724)\end{array}$ & -ve \\
\hline
\end{tabular}

1884 Asian Pacific Journal of Cancer Prevention, Vol 16, 2015 
Table 1 (cont). Characteristics and Results of the Eligible Studies

\begin{tabular}{|c|c|c|c|c|c|c|c|c|c|c|c|}
\hline $\mathrm{Li}$ & 2005 & China & 126 & NSCLC & I-IV & IHC & $\mathrm{CS}$ & $\begin{array}{l}\text { VEGFA } \\
77.8 \%\end{array}$ & Surv.curves & $\begin{array}{c}1.81 \\
(1.01-3.27)\end{array}$ & $+\mathrm{ve}$ \\
\hline Kim & 2005 & USA & 74 & NSCLC & I-II & IHC & $\mathrm{CS}$ & $\begin{array}{l}\text { VEGFA } \\
71.6 \%\end{array}$ & DE & $\begin{array}{c}2.147 \\
(0.939-4.907)\end{array}$ & $+\mathrm{ve}^{*}$ \\
\hline Huang & 2005 & Japan & $\begin{array}{l}97 \\
76\end{array}$ & NSCLC & $\begin{array}{l}\text { I } \\
\text { II-III }\end{array}$ & IHC & $\mathrm{CS}$ & $\begin{array}{c}\text { VEGFA } \\
48.5 \% \\
\text { VEGFC } \\
40.2 \% \\
\text { VEGFA } \\
44.6 \% \\
\text { VEGFC } \\
34.0 \%\end{array}$ & Report & $\begin{array}{c}2.37 \\
(1.07-5.24) \\
2.10 \\
(1.01-4.37) \\
1.27 \\
(0.69-2.35) \\
0.94 \\
(0.54-1.64)\end{array}$ & $\begin{array}{l}+\mathrm{ve} \\
+\mathrm{ve} \\
-\mathrm{ve} \\
-\mathrm{ve}\end{array}$ \\
\hline Nishi & 2005 & Japan & 100 & $\mathrm{ADC}$ & I-III & IHC & NR & $\begin{array}{l}\text { VEGFA } \\
22 \%\end{array}$ & Surv.curves & $\begin{array}{c}2.71 \\
(1.41-5.21)\end{array}$ & $+\mathrm{ve}$ \\
\hline Kojima & 2005 & Japan & 129 & $\mathrm{ADC}$ & I & IHC & $\mathrm{CS}$ & $\begin{array}{c}\text { VEGFA } \\
45.7 \% \\
\text { VEGFC } \\
43.4 \% \\
\text { VEGFR3 } \\
56.6 \% \\
\text { C+R3: } \\
31.8 \%\end{array}$ & Report & $\begin{array}{c}2.011 \\
(0.942-4.293) \\
1.646 \\
(0.760-3.565) \\
1.494 \\
(0.602-3.705) \\
2.513 \\
(1.315-4.801)\end{array}$ & $\begin{array}{l}-v e \\
-v e \\
-v e \\
+v e\end{array}$ \\
\hline Zhang L & 2004 & China & 43 & NSCLC & I-III & $\mathrm{IHC}$ & 10 & $\begin{array}{l}\text { VEGFA } \\
65.1 \%\end{array}$ & Surv.curves & $\begin{array}{c}2.90 \\
(1.04-8.07)\end{array}$ & $+\mathrm{ve}$ \\
\hline Yi & 2004 & China & 84 & NSCLC & I-IV & $\mathrm{IHC}$ & 10 & $\begin{array}{c}\text { VEGFC } \\
55.9 \% \\
\text { R3 } 59.5 \%\end{array}$ & Report & $\begin{array}{c}1.005 \\
(0.593-1.703) \\
3.370 \\
(1.813-6.265)\end{array}$ & $\begin{array}{l}-\mathrm{ve} \\
+\mathrm{ve}\end{array}$ \\
\hline Tanaka & 2004 & Japan & 162 & NSCLC & I & $\mathrm{IHC}$ & CS & $\begin{array}{l}\text { VEGFA } \\
38.9 \%\end{array}$ & Report & $\begin{array}{c}1.644 \\
(0.815-3.314)\end{array}$ & -ve \\
\hline Ogawa & 2004 & Japan & 125 & NSCLC & I-IIIA & IHC & $\mathrm{CS}$ & $\begin{array}{l}\text { VEGFC } \\
60.7 \%\end{array}$ & Report & $\begin{array}{c}1.724 \\
(1.087-2.734)\end{array}$ & $+\mathrm{ve}$ \\
\hline Nakashima & 2004 & Japan & $\begin{array}{c}153 \\
85 \\
52\end{array}$ & $\begin{array}{l}\text { NSCLC } \\
\text { ADC } \\
\text { SCC }\end{array}$ & I-IIIB & IHC & 30 & $\begin{array}{c}\text { VEGFA } \\
51.0 \% \\
\text { VEGFC } \\
41.8 \% \\
\text { VEGFA } \\
54.1 \% \\
\text { VEG- } \\
\text { FC45.9\% } \\
\text { VEGFA } \\
51.0 \% \\
\text { VEGFC } \\
40.4 \% \\
\end{array}$ & $\mathrm{DE}$ & $\begin{array}{c}2.012 \\
(1.181-3.427) \\
1.460 \\
(0.898-2.373) \\
3.816 \\
(1.602-9.092) \\
2.024 \\
(0.970-4.224) \\
0.913 \\
(0.319-2.611) \\
3.946 \\
(1.316-11.835)\end{array}$ & $\begin{array}{l}\text { +ve } \\
\text {-ve } \\
+\mathrm{ve} \\
\text {-ve } \\
\text {-ve } \\
+\mathrm{ve}\end{array}$ \\
\hline Mineo & 2004 & Italy & 51 & NSCLC & Ib-IIa & IHC & 25 & $\begin{array}{l}\text { VEGFA } \\
82.4 \%\end{array}$ & Report & $\begin{array}{c}3.617 \\
(1.054-14.610)\end{array}$ & $+\mathrm{ve}$ \\
\hline $\mathrm{Li}$ & 2004 & China & 96 & NSCLC & I-IIIB & IHC & $\mathrm{CS}$ & $\begin{array}{l}\text { VEGFA } \\
64.6 \%\end{array}$ & Surv.curves & $\begin{array}{c}1.82 \\
(1.01-3.29)\end{array}$ & $+\mathrm{ve}$ \\
\hline Iwasaki & 2004 & Japan & 71 & NSCLC & I-III & $\begin{array}{l}\text { ELI- } \\
\text { SA }\end{array}$ & $18 \mathrm{ng} /$ & VEGFA $39 \%$ & Report & $\begin{array}{c}2.060 \\
(1.024-4.778)\end{array}$ & $+\mathrm{ve}$ \\
\hline Saad & 2004 & USA & 50 & $\mathrm{ADC}$ & I & IHC & 20 & VEGFA $66 \%$ & Surv.curves & $\begin{array}{c}3.37 \\
(1.39-8.16)\end{array}$ & $+\mathrm{ve}$ \\
\hline Liang & 2003 & China & 55 & NSCLC & I-IV & IHC & 30 & $\begin{array}{l}\text { VEGFA } \\
74.6 \%\end{array}$ & Surv.curves & $\begin{array}{c}1.63 \\
(1.18-2.25)\end{array}$ & $+\mathrm{ve}$ \\
\hline $\mathrm{Li}$ & 2003 & China & 76 & NSCLC & I-IV & IHC & 10 & $\begin{array}{l}\text { VEGFC } \\
72.4 \%\end{array}$ & Surv.curves & $\begin{array}{c}2.55 \\
(1.22-5.34)\end{array}$ & $+\mathrm{ve}$ \\
\hline
\end{tabular}


Chun-Long Zheng et al

Table 1 (cont). Characteristics and Results of the Eligible Studies

\begin{tabular}{|c|c|c|c|c|c|c|c|c|c|c|c|}
\hline Arinaga & 2003 & Japan & 180 & NSCLC & I-III & IHC & 30 & $\begin{array}{c}\text { VEGFC } \\
76.1 \% \\
\text { VEGFR3: } \\
22 \% \\
\text { C+R3: } 22 \%\end{array}$ & $\begin{array}{l}\text { DC } \\
\text { Report } \\
\text { Surv.curves }\end{array}$ & $\begin{array}{c}1.554 \\
(0.989-2.442) \\
1.270 \\
(0.993-1.620) \\
2.32 \\
(1.03-5.24)\end{array}$ & $\begin{array}{l}\text {-ve } \\
+\mathrm{ve} * \\
+\mathrm{ve}\end{array}$ \\
\hline Ito & 2002 & Japan & 80 & $\mathrm{AC}$ & I-IV & IHC & 10 & VEGFA $40 \%$ & Surv.curves & $\begin{array}{c}1.30 \\
(0.20-8.32)\end{array}$ & -ve \\
\hline Tanaka & 2002 & Japan & 236 & NSCLC & I-III & IHC & CS & VEGFA $34 \%$ & Report & $\begin{array}{c}1.380 \\
(0.788-2.417)\end{array}$ & -ve \\
\hline Kojima & 2002 & Japan & $\begin{array}{l}94 \\
38\end{array}$ & $\begin{array}{l}\text { ADC } \\
\text { SCC }\end{array}$ & I & IHC & $\mathrm{CS}$ & $\begin{array}{l}\text { VEGFA } \\
60.6 \% \\
\text { VEGFA } \\
60.5 \%\end{array}$ & Report & $\begin{array}{c}3.374 \\
(0.911-12.488) \\
1.541 \\
(0.518-4.588)\end{array}$ & $\begin{array}{l}-\mathrm{ve} \\
-\mathrm{ve}\end{array}$ \\
\hline Inoshima & 2002 & Japan & 132 & NSCLC & I-IV & IHC & CS & $\begin{array}{l}\text { VEGFA } \\
49.2 \%\end{array}$ & Report & $\begin{array}{c}2.327(1.260- \\
4.262)\end{array}$ & $+\mathrm{ve}$ \\
\hline Minami & 2002 & Japan & 47 & $\mathrm{ADC}$ & I & IHC & 30 & $\begin{array}{l}\text { VEGFA } \\
29.8 \%\end{array}$ & Report & $\begin{array}{c}0.834(0.386- \\
1.371)\end{array}$ & $-v e$ \\
\hline Shou & 2001 & Japan & 119 & NSCLC & I-III & IHC & NR & $\begin{array}{l}\text { VEGFA } \\
63.9 \% \\
\end{array}$ & Surv.curves & $\begin{array}{c}1.77(0.92- \\
3.41) \\
\end{array}$ & $-\mathrm{ve}$ \\
\hline Osaki & 2001 & Japan & 33 & SCC & NR & IHC & NR & $\begin{array}{l}\text { VEGFA } \\
36.4 \%\end{array}$ & Surv.curves & $\begin{array}{c}4.56 \\
(1.15-18.13)\end{array}$ & $+\mathrm{ve}$ \\
\hline Niklinska & 2001 & Poland & 89 & NSCLC & I-IIIa & IHC & 70 & $\begin{array}{l}\text { VEGFA } \\
20 \% \\
\end{array}$ & Report & $\begin{array}{c}2.56 \\
(1.01-6.49) \\
\end{array}$ & $+\mathrm{ve}$ \\
\hline Masuya & 2001 & Japan & $\begin{array}{c}104 \\
34 \\
59\end{array}$ & $\begin{array}{l}\text { NSCLC } \\
\text { SCC } \\
\text { ADC }\end{array}$ & I-IIIB & IHC & 30 & $\begin{array}{l}\text { VEGFA } \\
51.9 \%\end{array}$ & DE & $\begin{array}{c}1.944 \\
(1.028-3.678) \\
9.730 \\
(2.298-41.194) \\
0.819 \\
(0.322-2.083) \\
\end{array}$ & $\begin{array}{l}+\mathrm{ve} \\
+\mathrm{ve} \\
-\mathrm{ve}\end{array}$ \\
\hline Liao & 2001 & China & 127 & NSCLC & I-III & IHC & 25 & $\begin{array}{l}\text { VEGFA } \\
40.9 \%\end{array}$ & Report & $\begin{array}{c}1.176 \\
(0.687-2.013)\end{array}$ & -ve \\
\hline Kang & 2001 & Korea & 61 & NSCLC & I-IIIB & IHC & 25 & $\begin{array}{l}\text { VEGFA } \\
88.5 \%\end{array}$ & Report & $\begin{array}{c}1.083 \\
(0.324-3.626) \\
\end{array}$ & -ve \\
\hline Kajita & 2001 & Japan & 62 & NSCLC & I-IV & IHC & 5 & $\begin{array}{c}\text { VEGFC } \\
38.7 \%\end{array}$ & Report & $\begin{array}{c}2.24 \\
(0.860-5.830) \\
\end{array}$ & -ve \\
\hline Han & 2001 & USA & 85 & NSCLC & I & IHC & 20 & $\begin{array}{l}\text { VEGFA } \\
71 \%\end{array}$ & Report & $\begin{array}{c}1.82 \\
(1.19-4.33)\end{array}$ & $+\mathrm{ve}$ \\
\hline Yano & 2000 & Japan & 108 & NSCLC & I-IV & IHC & 25 & $\begin{array}{c}\text { VEGFA } \\
45 \%\end{array}$ & $\mathrm{DE}$ & $\begin{array}{c}0.487 \\
(0.229-1.037) \\
\end{array}$ & -ve \\
\hline Sheng & 2000 & Japan & $\begin{array}{l}98 \\
57 \\
31\end{array}$ & $\begin{array}{l}\text { NSCLC } \\
\text { ADC } \\
\text { SCC }\end{array}$ & I-IV & IHC & 5 & $\begin{array}{c}\text { VEGFA } \\
51 \% \\
\text { VEGFA } \\
49.1 \% \\
\text { VEGFA } \\
61.3 \% \\
\end{array}$ & Surv.curves & $\begin{array}{c}3.27 \\
(1.41-7.67) \\
5.12 \\
(1.02-25.76) \\
4.86 \\
(1.02-22.86) \\
\end{array}$ & $\begin{array}{l}\text { +ve } \\
+\mathrm{ve} \\
+\mathrm{ve}\end{array}$ \\
\hline Ohta & 2000 & Japan & 122 & NSCLC & I & IHC & 10 & $\begin{array}{l}\text { VEGFC } \\
45.1 \%\end{array}$ & Report & $\begin{array}{c}1.34 \\
(0.58-3.10)\end{array}$ & -ve \\
\hline Odaka & 2000 & Japan & 67 & NSCLC & I & IHC & 20 & $\begin{array}{l}\text { VEGFA } \\
38.8 \% \\
\end{array}$ & $\mathrm{DE}$ & $\begin{array}{c}10.744 \\
(2.386-48.660) \\
\end{array}$ & $+\mathrm{ve}$ \\
\hline O’Byrne & 2000 & UK & 223 & NSCLC & I-III & IHC & 70 & $\begin{array}{l}\text { VEGFA } \\
46.6 \% \\
\end{array}$ & $\mathrm{DE}$ & $\begin{array}{c}1.63 \\
(1.080-2.460) \\
\end{array}$ & $+\mathrm{ve}$ \\
\hline Konishi & 2000 & Japan & 181 & NSCLC & $\begin{array}{l}\text { I- } \\
\text { IIIB }\end{array}$ & IHC & $\mathrm{CS}$ & $\begin{array}{l}\text { VEGFA } \\
25.4 \%\end{array}$ & Report & $\begin{array}{c}1.771 \\
(1.080-2.905) \\
\end{array}$ & $+\mathrm{ve}$ \\
\hline Oshika & 1998 & Japan & $\begin{array}{l}84 \\
25\end{array}$ & $\begin{array}{l}\text { NSCLC } \\
\text { SCC }\end{array}$ & NR & IHC & NR & $\begin{array}{c}\text { VEGFA } 40 \% \\
\text { VEGFA } \\
32.0 \%\end{array}$ & Surv.curves & $\begin{array}{c}3.95 \\
(1.29-12.12) \\
3.17 \\
(1.33-7.53)\end{array}$ & $\begin{array}{l}+\mathrm{ve} \\
+\mathrm{ve}\end{array}$ \\
\hline
\end{tabular}

1886 Asian Pacific Journal of Cancer Prevention, Vol 16, 2015 
Table 1 (cont). Characteristics and Results of the Eligible Studies

\begin{tabular}{|c|c|c|c|c|c|c|c|c|c|c|c|}
\hline Imoto & 1998 & Japan & $\begin{array}{l}91 \\
34\end{array}$ & $\begin{array}{l}\text { NSCLC } \\
\text { SCC }\end{array}$ & I-III & IHC & 5 & $\begin{array}{c}\text { VEGFA } 53 \% \\
\text { VEGFA } \\
38.2 \%\end{array}$ & $\begin{array}{l}\text { Report } \\
\text { Surv.curves }\end{array}$ & $\begin{array}{c}2.590 \\
(1.349-4.973) \\
3.67 \\
(1.91-7.07) \\
\end{array}$ & $\begin{array}{l}\text { +ve } \\
+\mathrm{ve}\end{array}$ \\
\hline $\begin{array}{l}\text { G i a t r o- } \\
\text { manola }\end{array}$ & 1998 & Greece & 114 & NSCLC & I-III & IHC & NR & VEGFA $68 \%$ & Surv.curves & $\begin{array}{c}1.56 \\
(1.02-2.38)\end{array}$ & $+\mathrm{ve}$ \\
\hline Shibusa & 1998 & Japan & 44 & $\mathrm{ADC}$ & I & IHC & CS & $\begin{array}{l}\text { VEGFA } \\
61.3 \%\end{array}$ & Surv.curves & $\begin{array}{c}3.65 \\
(1.01-13.25)\end{array}$ & $+\mathrm{ve}$ \\
\hline Volm & 1997 & $\begin{array}{l}\text { Ger- } \\
\text { many }\end{array}$ & 109 & SCC & I-IIIa & IHC & CS & $\begin{array}{c}\text { VEGFA 59\% } \\
\text { flt-1 } 68 \%\end{array}$ & Surv.curves & $\begin{array}{c}2.76(1.18- \\
6.40) \\
1.14(0.54-2.41)\end{array}$ & $\begin{array}{l}+\mathrm{ve} \\
-\mathrm{ve}\end{array}$ \\
\hline Fontanin & 1997 & Italy & 104 & NSCLC & I-III & IHC & 40 & VEGFA $44 \%$ & Surv.curves & $\begin{array}{c}2.61(1.01- \\
\quad 6.73)\end{array}$ & $+\mathrm{ve}$ \\
\hline Takanami & 1997 & Japan & 118 & $\mathrm{ADC}$ & I-IV & IHC & NR & VEGFA $58 \%$ & Report & $\begin{array}{c}1.54(0.91- \\
2.67)\end{array}$ & $+\mathrm{ve}^{*}$ \\
\hline
\end{tabular}

*VEGF,vascular endothelial growth factor. NSCLC,non-small cell lung cancer; adenocarcinoma; AC, adenocarcinoma; SCC, squamous cell carcinoma. IHC, immunohistochemistry; ELISA, enzyme linked immunosorbent assay. NR, not reported. CS, complex score combining intensity and percentage. OS, overall survival; RFS, recurrence-free survival; DFS, disease free survival; DSS, disease-specific survival. DE: data extrapolated; DC, data of -/+ convert +/- .Results, author's results; Positive, there was an inverse relationship between VEGFs and survival(poor prognosis); Inverse, there was a direct relationship between VEGFs and survival(good prognosis); -ve, there is no relationship. *,marginal value

represent significant statistically publication bias). All calculations were performed using STATA version 12.0 (Stata Corp., College Station, TX, USA).

\section{Results}

\section{Literature search}

The results of the literature research were presented in Figure 1. The initial search strategy identified 2470 potential relevant studies. 2302 of which were excluded after the initial review of their titles and abstracts: 633 articles duplicated; 767 had no direct link with the main subject (i.e. other tumors, indicators or not relevant articles); 392 of them were reviews and editorials; 423 articles of them were drug treatment, and 87 were case reports or case series. Then 168 full manuscripts were retrieved for detailed evaluation. 94 studies were excluded because of the lack of sufficient data $(n=36)$, serum level $(n=43)$, molecular level $(n=13)$ and duplicated $(n=2)$. Finally, 74 studies (Fontanini et al., 1997; Takanami et al., 1997; Volm et al., 1997; Giatromanolaki et al., 1998; Imoto et al., 1998; Oshika et al., 1998; Shibusa et al., 1998; Konishi et al., 2000; O’Byrne et al., 2000; Odaka et al., 2000; Ohta et al., 2000; Sheng et al., 2000; Yano et al., 2000; Han et al., 2001; Kajita et al., 2001; Kang et al., 2001; Liao et al., 2001; Masuya et al., 2001; Niklinska et al., 2001; Osaki et al., 2001; Shou et al., 2001; Inoshima et al., 2002; Ito et al., 2002; Kojima et al., 2002; Minami et al., 2002; Tanaka et al., 2002; Arinaga et al., 2003; Li et al., 2003; Liang et al., 2003; Iwasaki et al., 2004; Li et al., 2004; Mineo et al., 2004; Nakashima et al., 2004; Ogawa et al., 2004; Saad et al., 2004; Tanaka et al., 2004; Yi and Pan, 2004; Zhang et al., 2004; Huang et al., 2005; Kim et al., 2005; Kojima et al., 2005; Li et al., 2005; Nishi et al., 2005; Renyi-Vamos et al., 2005; Tomita et al., 2005; Enatsu et al., 2006; Seto et al., 2006; Zhang et al., 2006; Donnem et al., 2007; Maekawa et al., 2007; Ohta et al., 2007; Saintigny et al., 2007; Yilmaz et al., 2007; Yoo et

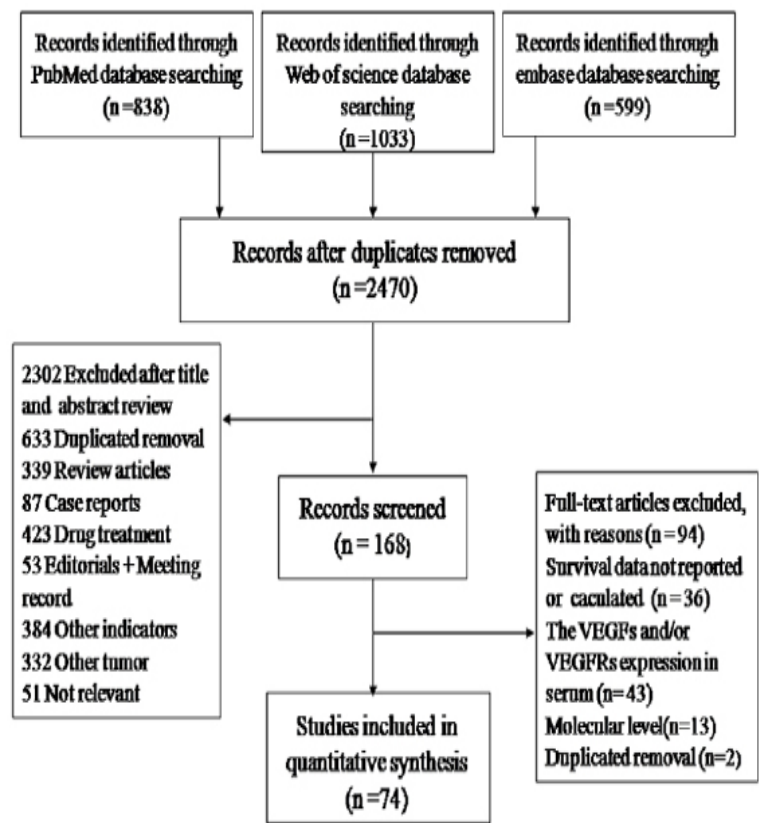

Figure 1. Flow Chart of Review Relevant Article for Meta-analysis

al., 2007; Zhou et al., 2007; Kadota et al., 2008; Ko et al., 2008; Bonnesen et al., 2009; Carrillo de Santa Pau et al., 2009; Sun et al., 2009; Bircan et al., 2010; Chen et al., 2010; Lin et al., 2010; Rades et al., 2010; Yamashita et al., 2010; Anagnostou et al., 2011; Chen et al., 2011; Dai et al., 2011; Donnem et al., 2011; Ucvet et al., 2011; Starnes et al., 2012; Wang et al., 2012; Yurdakul et al., 2012; Kim et al., 2013b) published between 1997 and 2014 were included according to the inclusion and exclusion criteria.

\section{Baseline characteristics}

The main characteristics of the studies included in the meta-analysis were shown in Table 1. A total 74 studies comprising 7631 patients were included in this meta-analysis. All studies reported the prognostic value 
Chun-Long Zheng et al

of VEGFs and/or VEGFRs status for survival in NSCLC patients, which total number of patients included was 7631 , ranging from 33 to 335 patients per study (median, 98). These publications fall into several different patient cohorts: 61 studies dealt with all types of NSCLC, 12

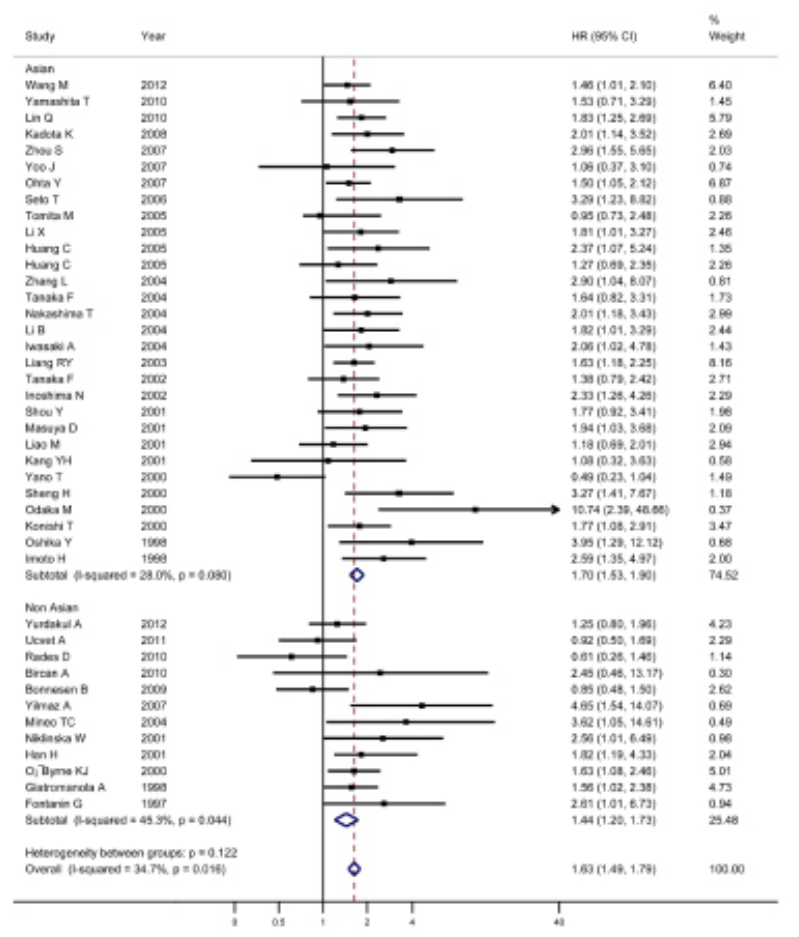

Figure 2. Hazard Ratio for Overall Survival of NSCLC Patients Stratified by Ethnicity with VEGFA Expression

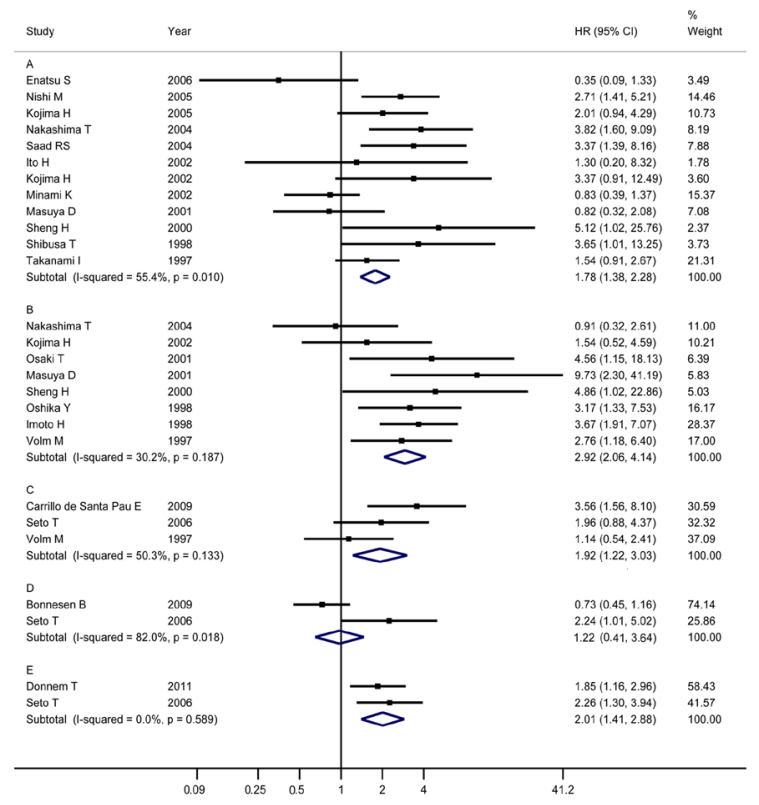

Figure 3. Hazard Ratio for Overall Survival of Different Patients with VEGFs or VEGFRs Expression. A) Hazard ratio of VEGFA expression in ADC; B) A) Hazard ratio of VEGFA expression in SCC; $\mathbf{C}$ ) Hazard ratio of VEGFR1 expression in NSCLC; D) Hazard ratio of VEGFR2 expression in NSCLC; $\mathbf{E}$ ) Hazard ratio of VEGFA/VEGFR2 co-expression in NSCLC studies dealt with ADC, 8 studies dealt with SCC. In all patients with NSCLC, surgery was performed as the

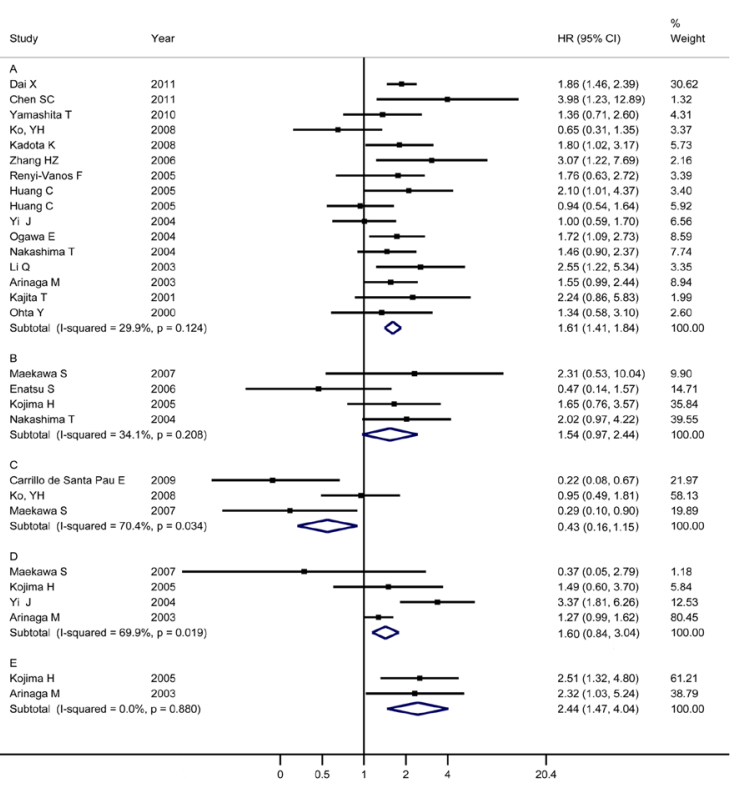

Figure 4. Hazard Ratio for Overall Survival of NSCLC Patients with VEGFC Expression. A) Hazard ratio of VEGFC expression in NSCLC; B) Hazard ratio of VEGFC expression in ADC;C) Hazard ratio of VEGFRD expression in NSCLC; D) Hazard ratio of VEGFR3 expression in NSCLC; E) Hazard ratio of VEGFC/VEGFR3 co-expression in NSCLC

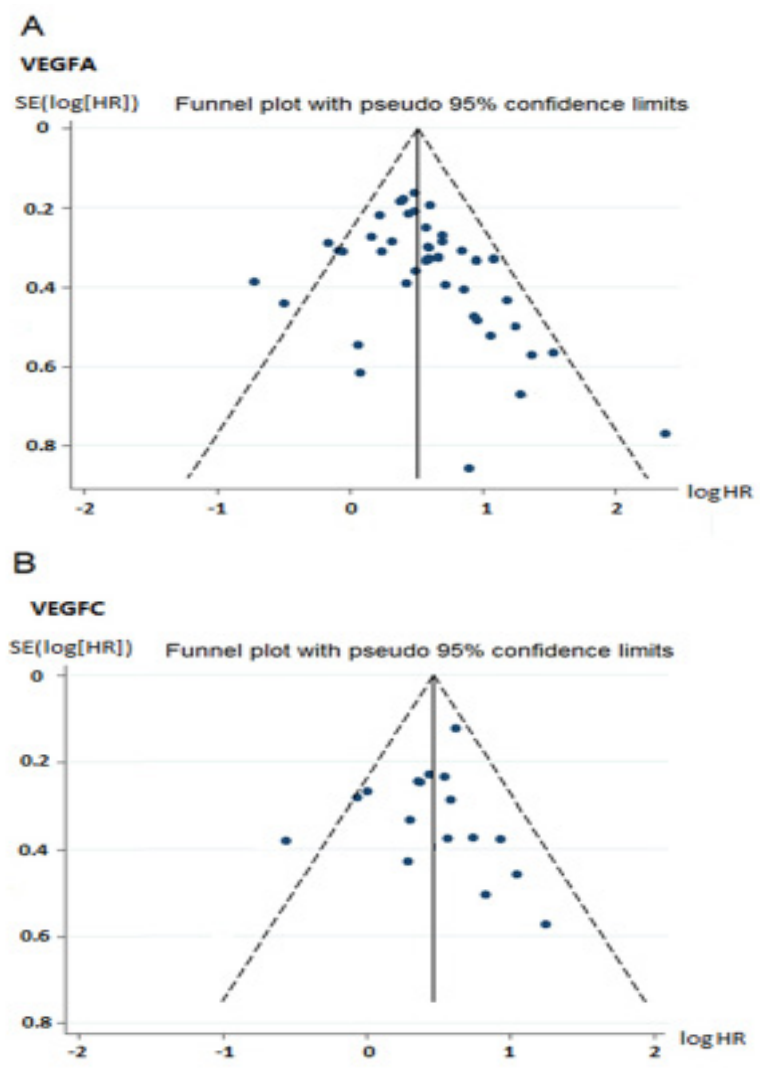

Figure 5. Funnel Plots Showing Publication Bias. A) Publication bias for VEGFA expression in NSCLC, $p=0.006$ (Begg's), $p=0.045$ (Egger's); B) Publication bias for VEGFC expression in NSCLC, $p=0.344$ (Begg's), $p=0.996$ (Egger's) 
foremost treatment measure. Seventy-two studies used IHC and two studies used ELISA to determine VEGFs and/ or VEGFRs expression. Among the 41 studies evaluating VEGFA expression in NSCLC, 29 studies (3525 patients, $75.68 \%$ ) were performed in Asian populations, the remaining 12 studies (1133 patients, 24.32\%) followed European or American patients. The proportion of patients exhibiting VEGFA expression in individual studies ranged from 20 to $96.1 \%$ by IHC. The rest of VEGFC, VEGFD, VEGFR1, VEGFR2 and VEGFR3 were detected in 20, 5, 4, 4 and 7 studies, respectively (Table 2).

Angiogenesis (VEGFA, VEGFR1, VEGFR2) and prognosis

The combined HR of VEGFA expression in NSCLC $(\mathrm{n}=41)$ was recorded as 1.633 (95\%CI: 1.490-1.791, $\mathrm{Q}=62.76, p=0.016, \mathrm{I}^{2}=34.7 \%$, Figure 2 ), indicating that positive immunostaining for VEGFA was significantly associated with adverse survival in the pooled patient group overall. When grouped according to the territorial scope of each studies, 1.704 (95\%CI: 1.532-1.896) in Asian and 1.443 (95\%CI: 1.202-1.732) in non-Asian (Table 2); When grouped according to the stage of NSCLC, the pooled HR was 1.887 (95\%CI: 1.554-2.292), 1.552 (95\% CI:1.373-1.754), 1.683 (95\%CI: 1.165-2.431) for stage I, I-III, I-IV, respectively (Table 2). The combined HR of stage I (1.887) was larger than stage I-III (1.552) and stage I-IV (1.683), suggesting that VEGFA expression could be an important prognostic factor for early stage NSCLC.

Studies evaluating VEGFA levels were separate aggregated to ADC and SCC for subgroup analyses, A statistically significantly correlation with survival was observed, particularly in SCC. The pooled HR was 1.775 (95\%CI: $1.384-2.275, \mathrm{Q}=24.65, p=0.010, \mathrm{I}^{2}=55.4 \%$, Figure 3A) in ADC ( $\mathrm{n}=12$,), whereas 2.919 (95\%CI: 2.060-4.137, $\mathrm{Q}=10.03, p=0.187, \mathrm{I}^{2}=30.2 \%$, Figure $3 \mathrm{~B}$ ) in $\operatorname{SCC}(\mathrm{n}=8)$.

With regard to the effects of the VEGFR1 expression on survival in NSCLC $(n=4)$, the pooled HR was 1.745 (95\% CI: 1.339-2.274, $\mathrm{Q}=4.30, p=0.231, \mathrm{I}^{2}=30.2 \%$, Table 2), and taken the one DSS included out $(n=3)$, the combined HR was 1.924 (95\%CI: 1.220-3.034, Q=4.03, $p=0.133, \mathrm{I}^{2}=50.3 \%$, Figure $3 \mathrm{C}$ ), suggesting that VEGFR 1 high expression is significantly associated with low survival rates.

Interestingly, highly significant statistically influence of VEGFA/VEGFR2 co-expression on survival in NSCLC patients was detected, with the combined HR was 2.011 (95\%CI: $1.405-2.876, \mathrm{Q}=0.29, p=0.589, \mathrm{I}^{2}=0.0 \%$, Figure $3 \mathrm{E})$. However, statistically significant effect of VEGFR2 expression on survival wasn't observed, the pooled HR was $1.270(95 \% \mathrm{CI}: 0.793-2.034, \mathrm{Q}=8.13, p=0.043$, $\mathrm{I}^{2}=63.10 \%$, Table 2) for overall, and 1.218 (95\%CI: 0.4083.640, $\mathrm{Q}=5.57, p=0.018, \mathrm{I}^{2}=82.0 \%$, Figure 3D) for OS.

\section{Lymphangiogenesis (VEGFC, VEGFD, VEGFR3) and prognosis}

The pooled results from the 20 studies evaluating VEGFC expression in NSCLC was 1.609 (95\%CI: 1.4201.823, $\mathrm{Q}=25.29, p=0.191, \mathrm{I}^{2}=20.9 \%$, Table 2), and 1.611 (95\%CI: $1.407-1.844, \mathrm{Q}=21.41, p=0.124, \mathrm{I}^{2}=29.9 \%$,
Figure 4A) when five non-OS excluded, indicating that VEGFC expression was an indicator of poor prognosis for NSCLC patients. When the four studies investigating VEGFC expression limited to the patients with ADC, which statistically significant effect on survival wasn't observed, with the pooled HR 1.536 (95\%CI: 0.967-2.440, $\mathrm{Q}=4.55, p=0.208, \mathrm{I}^{2}=34.1 \%$, Figure $\left.4 \mathrm{~B}\right)$.

The combined HR of seven eligible studies evaluating VEGFR3 expression in NSCLC was 1.513 (95\%CI: 1.267$1.808, \mathrm{Q}=11.10, p=0.085, \mathrm{I}^{2}=46 \%$, Table 2$)$, and when three non-OS excluded, the pooled HR was 1.596 (95\%CI: 0.837-3.045, $\mathrm{Q}=9.97, p=0.019, \mathrm{I}^{2}=69.9 \%$, Figure 4D), which statistically significant effect on survival wasn't detected. In addition, the data collected was not sufficient to determine the prognostic value of VEGFC in patients with ADC and SCC, separately.

To our surprise is the VEGFC/VEGFR3 co-expression was highly significant prognostic value in NSCLC, with the pooled HR was 2.436 (95\%CI:1.468-4.043, Q=0.020, $p=0.880, \mathrm{I}^{2}=0.0 \%$, Figure $\left.4 \mathrm{E}\right)$.

With regard to the effects of the VEGFD expression on survival in NSCLC $(n=5)$, the pooled result was a marginal value $(\mathrm{HR}=0.596,95 \% \mathrm{CI}: 0.336-1.058, \mathrm{Q}=9.68$, $p=0.046, \mathrm{I}^{2}=58.7 \%$, Table 2$)$. When the two DSS excluded $(\mathrm{n}=3)$, the combined HR was 0.427 (95\%CI: 0.159-1.150, $\mathrm{Q}=6.75, p=0.034, \mathrm{I}^{2}=70.4 \%$, Figure $4 \mathrm{C}$ ). These results show that VEGFD expression was more likely associated with good outcome. Unfortunately, the more sufficient data were required to determine the prognostic value of VEGFD expression.

\section{Heterogeneity analysis}

This systematic review with meta-analysis was inspected by heterogeneity test. Highly significant heterogeneity was found among 8 studies of stage I-IV NSCLC with VEGFC expression, 4 studies of NSCLC with VEGFR2 expression, 5 studies of NSCLC with VEGFD expression, 7 studies of NSCLC with VEGFR3 expression (Table 2).

\section{Publication bias}

The publication bias in the literature was quantitative evaluated by Begg's and Egger's test. The absence of publication bias was found in 15 studies investigating VEGFC expression in patients with NSCLC, with a Begg's test score of $p=0.344$ and an Egger's test score of $p=0.996$ (Figure 5B), Similar results were found in the four studies with VEGFR1 expression ( $p=0.308$ and 0.671$)$, four studies including patients withVEGFR2 expression ( $p=0.734$ and 0.929$)$ and eight studies investigating VEGFR3 expression in patients with NSCLC $(p=0.764$ and 0.872).

However, the funnel plot revealed an apparent asymmetry in 41 eligible studies investigating NSCLC patients with VEGFA expression ( $p=0.006$ and 0.045$)$ (Figure 5A) and five studies investigating VEGFD expression ( $p=0.086$ and 0.004 ), suggesting the presence of a potential publication bias, The bias of publication could be explained by a language bias, inflated estimates by a flawed methodological design in smaller studies, what really counts is lack of publication of trials with opposite 


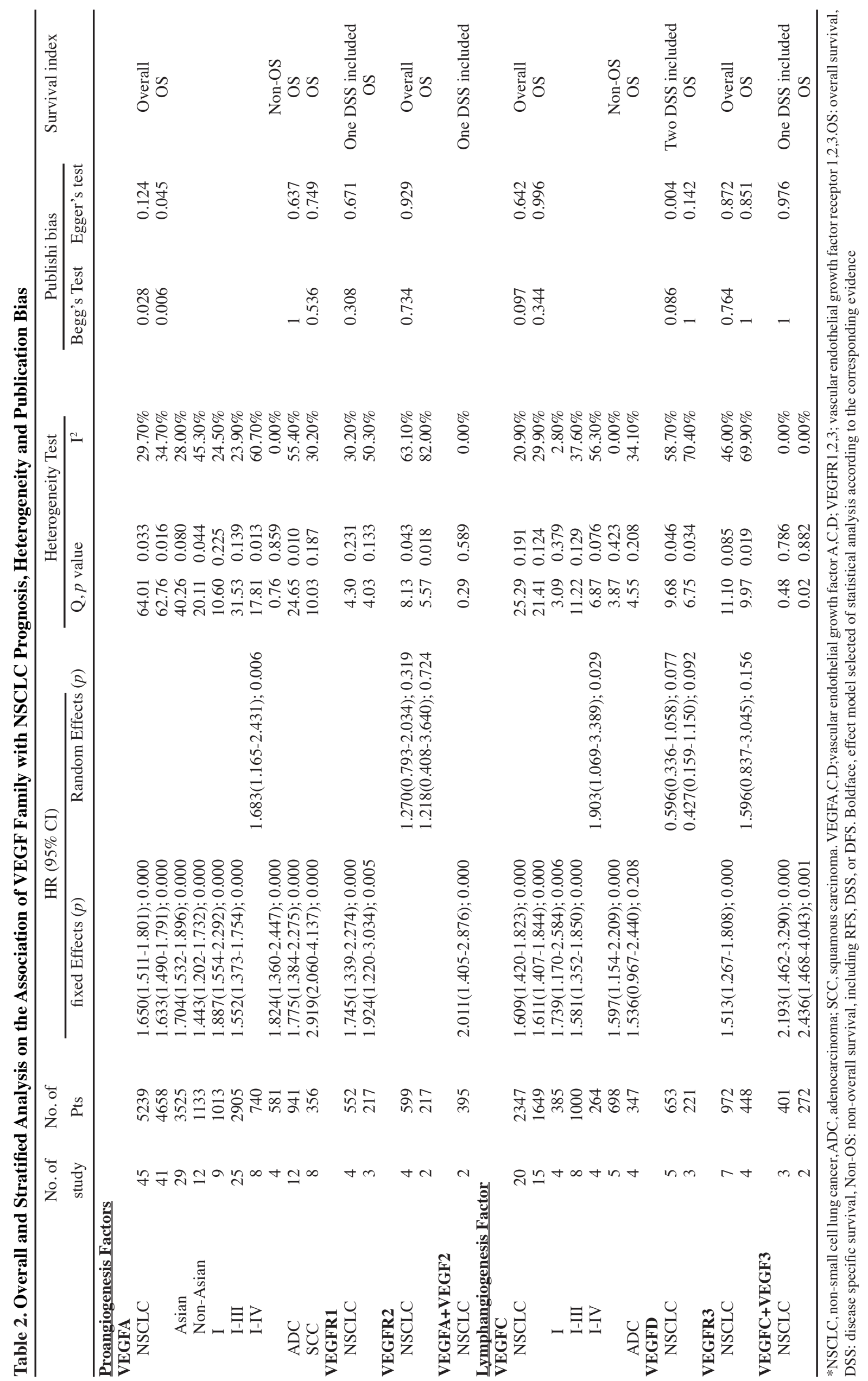

1890 Asian Pacific Journal of Cancer Prevention, Vol 16, 2015 
results (Table 2).

\section{Discussion}

Our meta-analysis showed that high VEGFs and/or VEGFRs expression did indeed predict poor survival in patients with NSCLC. For angiogenesis, our result clearly indicated that VEGFA expression has a significant correlation with poor survival in patients with NSCLC. When the analyses were restricted to the stages of NSCLC, VEGFA expression could be an important prognostic factor for early stage NSCLC ( $\mathrm{HR}=1.887$ for stage I). When the analyses were restricted to the histologies of NSCLC, a significant prognostic significance $(H R=2.919)$ was found in lung SCC patients. Data analysis revealed that VEGFR1 expression associated with low survival rate, but the VEGFR2 expression wasn't detected statistically significant effect on survival in NSCLC patients. For lymphangiogenesis, the expression of VEGFC predicted a poor prognosis in NSCLC patients. However, the VEGFC overexpression was only associated with poor survival. Unfortunately, neither the VEGFC expression nor the VEGFR3 expression was sufficient to determine the prognostic value in lung SCC patients.

The emergence of the targeted therapies for NSCLC has generated a need for accurate histologic subtyping of NSCLC (Kim et al., 2013a) because of the different clinicopathological and molecular characteristics of ADC and SCC (Miller et al., 2004; Inamura et al., 2010). Patients with SCC were not recommended to receive bevasizumab (Avastin) because of a $30 \%$ mortality rate due to fatal hemorrhage (Johnson et al., 2004; Cohen et al., 2007; Yan et al., 2011; Stead et al., 2012). There were very few previous literatures reported the prognostic impact in lung SCC patient with VEGFA expression. Our pooled analysis showed that VEGFA overexpression was associated with worse survival in patients with SCC (HR=2.919; 95\%CI: 2.060-4.137). Therefore, the severe bleeding may be a significant response. Several ongoing clinical randomized controlled trials do include squamous participants (Schiller et al., 2009; Spratlin et al., 2010; Sternberg et al., 2010; Doebele et al., 2012) in recent years. The role of angiogenesis inhibition in the adjuvant setting is currently being tested by the ECOG (Eastern Cooperative Oncology Group) 1505 trial, in which NSCLC patients (including $31 \%$ SCC) with completely resected tumors are randomly assigned to chemotherapy alone or in combination with bevacizumab. The safest setting to pursue further evaluation of bevacizumab in patients with SCC seems to be after surgical resection. Our conclusion supports the new viewpoint that antiVEGF therapies may be a reliable targeted therapy for postoperative SCC patients.

There were very few previous literatures reported the prognostic impact of VEGF/VEGFR co-expression in patients with NSCLC. Our meta-analysis explored the prognostic impact of VEGF/VEGFR co-expression on survival in patients with NSCLC. The combined HR of VEGFA/VEGFR2 co-expression in NSCLC was recorded as 2.011 (95\% CI: $1.405-2.876, \mathrm{Q}=0.29, p=0.589$, $\mathrm{I}^{2}=0.0 \%$ ), indicating that positive immunostaining for
VEGFA/VEGFR2 co-expression was significantly associated with adverse survival. The VEGFC/VEGFR3 co-expression also had highly significant prognostic value in NSCLC, with the pooled HR was 2.436 (95\%CI: $1.468-$ $\left.4.043, \mathrm{Q}=0.020, p=0.880, \mathrm{I}^{2}=0.0 \%\right)$. Empirically, HRs $>2$ are considered strongly predictive (Hayes et al., 2001). In a word, both VEGFA/VEGFR2 co-expression and VEGFC/ VEGFR3 co-expression were sufficient discriminate value as preferable prognostic biologic marker for an individual undergoing treatment.

Our data were consistent with the three previous meta-analysis (Delmotte et al., 2002; Zhan et al., 2009; Jiang et al., 2014), which separately included 15, 51, 16 studies. Besides, the previous analyses were insufficient to determine the prognostic value of VEGFD, VEGFRs and VEGF/VEGFR co-expression in NSCLC. We have improved these deficiencies by incorporating more related studies in recent years. What is more, we have found the several biologic markers (i.e. VEGFA expression in SCC, VEGFA/VEGFR2 co-expression in NSCLC, VEGFC/ VEGFR3 co-expression in NSCLC, etc.) with preferable discriminate value for an individual prognosis.

In addition, heterogeneity and potential publication bias were assessed in accordance with published guidelines. There were several potential sources of heterogeneity: the different of baseline characteristics of patients included (age, tumor size, and stage), the adjuvant treatment they might have received; the duration of follow-up, the differences in the cutoff value $(5 \%, 10 \%, 20 \%, 25 \%, 50 \%$, complex scores) of IHC method used, the distribution of immunostaining used for scoring was not explicitly stated in the text (i.e. cytoplasmic, membranous, nuclear, stromal, etc.), the primary antibody used wasn't identical, the different criteria used for immunohistochemical classification. But the Der Simonian and Laird method we used (random effect model) took them into account (DerSimonian and Laird, 1986).

The extract method of HRs and 95\% CI was one potential source of bias. The exact value of VEGF and/or VEGFRs expression status requires to be determined by appropriate multivariate analysis. Data for multivariate survival analysis reported were included in the present systematic review with meta-analysis; if these data were not available, we extrapolated them from the survival curves as univariate analysis incorporated, necessarily making assumptions about the censoring process. The choice of study and detected method was another potential source of bias. Firstly, the prognostic value should be confirmed by adequately designed prospective study. Unfortunately, few prospectively designed prognostic studies concerning biomarkers have been reported. Our collected many retrospective studies to reveal their clinical significance. Secondly, the variability of IHC method was an inconvenient source of selection bias. In future studies, the assessment of these prognostic factors should be better standard, especially for patients whom adjuvant therapy is recommended (Vermeulen et al., 2002).

The major concern for all forms of meta-analysis is publication bias (Dubben and Beck-Bornholdt, 2005). The present analysis found significant publication bias among 47 studies of NSCLC patients with VEGFA expression, 
which funnel plot revealed an apparent asymmetry. The bias of publication could be explained by a language bias, inflated estimates by a flawed methodological design in smaller studies. Nevertheless, the most important point is a lack of publication of trials with opposite results. We attempted to minimize publication bias by making our literature search as complete as possible, using three databases (PubMed, EMBASE and Web of Science). The various published studies, which conclusions were discrepancy, could have encouraged researchers to publish their data whether positive or negative, such publication bias will be limited.

In summary, the expression of VEGFA (particularly in SCC and early stage NSCLC), VEGFC and VEGFR1 indicates an unfavorable impact on survival of patients with NSCLC, respectively. However, the expression of VEGFD seems to have no significant impact on survival of NSCLC patients, and the VEGFR2 expression also wasn't associated with low survival rate in our meta-analysis. Furthermore, the co-expression of VEGFA/VEGFR2, VEGFC/VEGFR3 reveals sufficient discriminate value for an individual as preferable prognostic biologic markers. These results should be confirmed by adequately further study. The findings of this study will encourage more people to identify the VEGF/VEGFR co-expression as ideal prognostic indicators in clinical practice in the future.

\section{Acknowledgements}

The authors would like to thank all the investigators of the primary studies and all the members of our team.

\section{References}

Alberg AJ, Samet JM (2003). Epidemiology of lung cancer. Chest, 123, 21-49.

Alitalo K, Carmeliet P (2002). Molecular mechanisms of lymphangiogenesis in health and disease. Cancer Cell, 1, 219-27.

Anagnostou VK, Tiniakos DG, Fotinou M, et al (2011). Multiplexed analysis of angiogenesis and lymphangiogenesis factors predicts outcome for non-small cell lung cancer patients. Virchows Arch, 458, 331-40.

Arinaga M, Noguchi T, Takeno S, et al (2003). Clinical significance of vascular endothelial growth factor $\mathrm{C}$ and vascular endothelial growth factor receptor 3 in patients with nonsmall cell lung carcinoma. Cancer, 97, 457-64.

Begg CB, Mazumdar M (1994). Operating characteristics of a rank correlation test for publication bias. Biometrics, 50, 1088-101.

Bircan A, Bircan S, Kapucuoglu N, et al (2010). Maspin, VEGF and $\mathrm{p} 53$ expression in small biopsies of primary advanced lung cancer and relationship with clinicopathologic parameters. Pathol Oncol Res, 16, 553-61.

Bonnesen B, Pappot H, Holmstav J, et al (2009). Vascular endothelial growth factor A and vascular endothelial growth factor receptor 2 expression in non-small cell lung cancer patients: relation to prognosis. Lung Cancer, 66, 314-8.

Carrillo de Santa Pau E, Arias FC, Caso Pelaez E, et al (2009). Prognostic significance of the expression of vascular endothelial growth factors A, B, C, and D and their receptors $\mathrm{R} 1, \mathrm{R} 2$, and R3 in patients with nonsmall cell lung cancer. Cancer, 115, 1701-12.

Chen G, Liu XY, Wang Z, et al (2010). Vascular endothelial growth factor $\mathrm{C}$ : the predicator of early recurrence in patients with N2 non-small-cell lung cancer. Eur J Cardiothorac Surg, 37, 546-51.

Chen SC, Shih CM, Tseng GC, et al (2011). Vascular endothelial growth factor $\mathrm{C}$ as a predictor of early recurrence and poor prognosis of resected stage I non-small cell lung cancer. Ann Acad Med Singapore, 40, 319-24.

Cohen MH, Gootenberg J, Keegan P, et al (2007). FDA drug approval summary: bevacizumab (Avastin) plus Carboplatin and Paclitaxel as first-line treatment of advanced/metastatic recurrent nonsquamous non-small cell lung cancer. Oncologist, 12, 713-8.

Dai X, Wang W, Shen-Tu Y, et al (2011). Expression and prognostic value of VEGF-C and lymphangeogenesis in lung adenocarcinoma and squamous cell carcinoma. Zhongguo Fei Ai Za Zhi, 14, 774-9.

Delmotte P, Martin B, Paesmans M, et al (2002). The role of vascular endothelial growth factor in the survival of patients with lung cancer: a systematic literature review and metaanalysis. Revue des Maladies Respiratoires, 19, 577-84.

DerSimonian R, Laird N (1986). Meta-analysis in clinical trials. Control Clin Trials, 7, 177-88.

Doebele RC, Conkling P, Traynor AM, et al (2012). A phase I, open-label dose-escalation study of continuous treatment with BIBF 1120 in combination with paclitaxel and carboplatin as first-line treatment in patients with advanced non-small-cell lung cancer. Ann Oncol, 23, 2094-102.

Donnem T, Al-Saad S, Al-Shibli K, et al (2007). Inverse prognostic impact of angiogenic marker expression in tumor cells versus stromal cells in non small cell lung cancer. Clin Cancer Res, 13, 6649-57.

Donnem T, Andersen S, Al-Saad S, et al (2011). Prognostic impact of angiogenic markers in non-small-cell lung cancer is related to tumor size. Clin Lung Cancer, 12, 106-15.

Dubben HH, Beck-Bornholdt HP (2005). Systematic review of publication bias in studies on publication bias. $B M J, \mathbf{3 3 1}$, 433-4.

Dwyer T, Couper D, Walter SD (2001). Sources of heterogeneity in the meta-analysis of observational studies: the example of SIDS and sleeping position. J Clin Epidemiol, 54, 440-7.

Egger M, Davey Smith G, Schneider M, et al (1997). Bias in meta-analysis detected by a simple, graphical test. $B M J$, 315, 629-34.

Enatsu S, Iwasaki A, Shirakusa T, et al (2006). Expression of hypoxia-inducible factor-1 alpha and its prognostic significance in small-sized adenocarcinomas of the lung. Eur J Cardiothorac Surg, 29, 891-5.

Ferrara N (2002). VEGF and the quest for tumour angiogenesis factors. Nat Rev Cancer, 2, 795-803.

Ferrara N, Kerbel RS (2005). Angiogenesis as a therapeutic target. Nature, 438, 967-74.

Folkman J (1971). Tumor angiogenesis: therapeutic implications. N Engl J Med, 285, 1182-6.

Fontanini G, Vignati S, Boldrini L, et al (1997). Vascular endothelial growth factor is associated with neovascularization and influences progression of non-small cell lung carcinoma. Clin Cancer Res, 3, 861-5.

Giatromanolaki A, Koukourakis MI, Kakolyris S, et al (1998). Vascular endothelial growth factor, wild-type p53, and angiogenesis in early operable non-small cell lung cancer. Clin Cancer Res, 4, 3017-24.

Gimbrone MA, Leapman SB, Cotran RS, et al (1972). Tumor dormancy in vivo by prevention of neovascularization. $J$ Exp Med, 136, 261-76.

Han H, Silverman JF, Santucci TS, et al (2001). Vascular endothelial growth factor expression in stage I non-small cell lung cancer correlates with neoangiogenesis and a poor 
prognosis. Ann Surg Oncol, 8, 72-9.

Hardy RJ, Thompson SG (1998). Detecting and describing heterogeneity in meta-analysis. Stat Med, 17, 841-56.

Hayes DF, Isaacs C, Stearns V (2001). Prognostic factors in breast cancer: current and new predictors of metastasis. $J$ Mammary Gland Biol Neoplasia, 6, 375-92.

He Y, Rajantie I, Pajusola K, et al (2005). Vascular endothelial cell growth factor receptor 3-mediated activation of lymphatic endothelium is crucial for tumor cell entry and spread via lymphatic vessels. Cancer Res, 65, 4739-46.

Hicklin DJ, Ellis LM (2005). Role of the vascular endothelial growth factor pathway in tumor growth and angiogenesis. J Clin Oncol, 23, 1011-27.

Higgins JP, Thompson SG (2002). Quantifying heterogeneity in a meta-analysis. Stat Med, 21, 1539-58.

Huang C, Liu D, Masuya D, et al (2005). Clinical application of biological markers for treatments of resectable non-smallcell lung cancers. Br J Cancer, 92, 1231-9.

Imoto H, Osaki T, Taga S, et al (1998). Vascular endothelial growth factor expression in non-small-cell lung cancer: prognostic significance in squamous cell carcinoma. $J$ Thorac Cardiovasc Surg, 115, 1007-14.

Inamura K, Ninomiya H, Ishikawa Y, et al (2010). Is the epidermal growth factor receptor status in lung cancers reflected in clinicopathologic features? Arch Pathol Lab Med, 134, 66-72.

Inoshima N, Nakanishi Y, Minami T, et al (2002). The influence of dendritic cell infiltration and vascular endothelial growth factor expression on the prognosis of non-small cell lung cancer. Clin Cancer Res, 8, 3480-6.

Ito H, Oshita F, Kameda Y, et al (2002). Expression of vascular endothelial growth factor and basic fibroblast growth factor in small adenocarcinomas. Oncol Rep, 9, 119-23.

Iwasaki A, Kuwahara M, Yoshinaga Y, et al (2004). Basic fibroblast growth factor (bFGF) and vascular endothelial growth factor (VEGF) levels, as prognostic indicators in NSCLC. Eur J Cardiothorac Surg, 25, 443-8.

Jiang H, Shao W, Zhao W (2014). VEGF-C in non-small cell lung cancer: Meta-analysis. Clin Chim Acta, 427, 94-9.

Johnson DH, Fehrenbacher L, Novotny WF, et al (2004). Randomized phase II trial comparing bevacizumab plus carboplatin and paclitaxel with carboplatin and paclitaxel alone in previously untreated locally advanced or metastatic non-small-cell lung cancer. J Clin Oncol, 22, 2184-91.

Kadota K, Huang CL, Liu D, et al (2008). The clinical significance of lymphangiogenesis and angiogenesis in nonsmall cell lung cancer patients. Eur J Cancer, 44, 1057-67.

Kaira K, Yamamoto N (2010). Prognostic and predictive factors in resected non-small-cell lung cancer. Expert Opin Med Diagn, 4, 373-81.

Kajita T, Ohta Y, Kimura K, et al (2001). The expression of vascular endothelial growth factor $\mathrm{C}$ and its receptors in non-small cell lung cancer. Br J Cancer, 85, 255-60.

Kang YH, Kim KS, Yu YK, et al (2001). The relationship between microvessel count and the expression of vascular endothelial growth factor, p53, and K-ras in non-small cell lung cancer. J Korean Med Sci, 16, 417-23.

Kim MJ, Shin HC, Shin KC, et al (2013a). Best immunohistochemical panel in distinguishing adenocarcinoma from squamous cell carcinoma of lung: tissue microarray assay in resected lung cancer specimens. Ann Diagn Pathol, 17, 85-90.

Kim MS, Park TI, Lee YM, et al (2013b). Expression of Id-1 and VEGF in non-small cell lung cancer. Int J Clin Exp Pathol, 6, 2102-11.

Kim SJ, Rabbani ZN, Dewhirst MW, et al (2005). Expression of HIF-1alpha, CA IX, VEGF, and MMP-9 in surgically resected non-small cell lung cancer. Lung Cancer, $\mathbf{4 9 ,}$ 325-35.

Ko YH, Jung CK, Lee MA, et al (2008). Clinical significance of vascular endothelial growth factors (VEGF)-C and -D in resected non-small cell lung cancer. Cancer Res Treat, 40, $133-40$

Kojima H, Shijubo N, Abe S (2002). Thymidine phosphorylase and vascular endothelial growth factor in patients with Stage I lung adenocarcinoma. Cancer, 94, 1083-93.

Kojima H, Shijubo N, Yamada G, et al (2005). Clinical significance of vascular endothelial growth factor-C and vascular endothelial growth factor receptor 3 in patients with T1 lung adenocarcinoma. Cancer, 104, 1668-77.

Konishi T, Huang CL, Adachi M, et al (2000). The K-ras gene regulates vascular endothelial growth factor gene expression in non-small cell lung cancers. Int J Oncol, 16, 501-11.

Li B, Cao X, Zhang J, et al (2004). Expression and clinical significance of vascular endothelial growth factor in human non-small cell lung cancer. Zhongguo Fei Ai Za Zhi, 7, 142-5.

Li Q, Dong X, Gu W, et al (2003). Clinical significance of coexpression of VEGF-C and VEGFR-3 in non-small cell lung cancer. Chin Med J (Engl), 116, 727-30.

Li X, Li C, Zhang W, et al (2005). Expressions of cyclooxygenase2 and vascular endothelial growth factor in human lung cancer tissue. Zhongguo Fei Ai Za Zhi, 8, 112-5.

Liang RY, Liao ZS, Jiang SP, et al (2003). Expression of cyclin D1 and vascular endothelial growth factor(VEGF) in nonsmall cell lung carcinoma and their association with the prognosis. Ai Zheng, 22, 86-90.

Liao M, Wang H, Lin Z, et al (2001). Vascular endothelial growth factor and other biological predictors related to the postoperative survival rate on non-small cell lung cancer. Lung Cancer, 33, 125-32.

Lin Q, Li M, Shen ZY, et al (2010). Prognostic impact of vascular endothelial growth factor-A and E-cadherin expression in completely resected pathologic stage I non-small cell lung cancer. Jpn J Clin Oncol, 40, 670-6.

Maekawa S, Iwasaki A, Shirakusa T, et al (2007). Correlation between lymph node metastasis and the expression of VEGF-C, VEGF-D and VEGFR-3 in T1 lung adenocarcinoma. Anticancer Res, 27, 3735-41.

Masuya D, Huang C, Liu D, et al (2001). The intratumoral expression of vascular endothelial growth factor and interleukin-8 associated with angiogenesis in nonsmall cell lung carcinoma patients. Cancer, 92, 2628-38.

Miller VA, Kris MG, Shah N, et al (2004). Bronchioloalveolar pathologic subtype and smoking history predict sensitivity to gefitinib in advanced non-small-cell lung cancer. J Clin Oncol, 22, 1103-9.

Minami K, Saito Y, Imamura H, et al (2002). Prognostic significance of p53, Ki-67, VEGF and Glut-1 in resected stage I adenocarcinoma of the lung. Lung Cancer, 38, 51-7.

Mineo TC, Ambrogi V, Baldi A, et al (2004). Prognostic impact of VEGF, CD31, CD34, and CD105 expression and tumour vessel invasion after radical surgery for IB-IIA non-small cell lung cancer. J Clin Pathol, 57, 591-7.

Molina JR, Yang P, Cassivi SD, et al (2008). Non-small cell lung cancer: epidemiology, risk factors, treatment, and survivorship. Mayo Clin Proc, 83, 584-94.

Nakashima T, Huang CL, Liu D, et al (2004). Expression of vascular endothelial growth factor-A and vascular endothelial growth factor- $\mathrm{C}$ as prognostic factors for nonsmall cell lung cancer. Med Sci Monit, 10, 157-65.

Niklinska W, Burzykowski T, Chyczewski L, et al (2001). Expression of vascular endothelial growth factor (VEGF) in non-small cell lung cancer (NSCLC): association with p53 gene mutation and prognosis. Lung Cancer, 34, 59-64. 
Nishi M, Abe Y, Tomii Y, et al (2005). Cell binding isoforms of vascular endothelial growth factor-A (VEGF189) contribute to blood flow-distant metastasis of pulmonary adenocarcinoma. Int J Oncol, 26, 1517-24.

O’Byrne KJ, Koukourakis MI, Giatromanolaki A, et al (2000). Vascular endothelial growth factor, platelet-derived endothelial cell growth factor and angiogenesis in non-smallcell lung cancer. Br J Cancer, 82, 1427-32.

Odaka M, Sato S, Masubuchi M, et al (2000). Comparison of VEGF and 553 protein expression in stage I non-small cell lung cancer. Japanese J Lung Cancer, 40, 11-5.

Ogawa E, Takenaka K, Yanagihara K, et al (2004). Clinical significance of VEGF-C status in tumour cells and stromal macrophages in non-small cell lung cancer patients. $\mathrm{Br} J$ Cancer, 91, 498-503.

Ohta Y, Nozawa H, Tanaka Y, et al (2000). Increased vascular endothelial growth factor and vascular endothelial growth factor-c and decreased $\mathrm{nm} 23$ expression associated with microdissemination in the lymph nodes in stage I non-small cell lung cancer. J Thorac Cardiovasc Surg, 119, 804-13.

Ohta Y, Tanaka Y, Watanabe G, et al (2007). Predicting recurrence following curative surgery in stage I non-small cell lung cancer patients using an angiogenesis-associated factor. J Exp Clin Cancer Res, 26, 301-5.

Osaki T, Oyama T, Inoue M, et al (2001). Molecular biological markers and micrometastasis in resected non-small-cell lung cancer. prognostic implications. Jpn J Thorac Cardiovasc Surg, 49, 545-51.

Oshika Y, Nakamura M, Tokunaga T, et al (1998). Expression of cell-associated isoform of vascular endothelial growth factor 189 and its prognostic relevance in non-small cell lung cancer. Int J Oncol, 12, 541-4.

Paesmans M, Sculier JP, Libert P, et al (1995). Prognostic factors for survival in advanced non-small-cell lung cancer: univariate and multivariate analyses including recursive partitioning and amalgamation algorithms in 1,052 patients. the european lung cancer working party. J Clin Oncol, 13, 1221-30.

Peters JL, Sutton AJ, Jones DR, et al (2008). Contour-enhanced meta-analysis funnel plots help distinguish publication bias from other causes of asymmetry. J Clin Epidemiol, 61, 991-6.

Rades D, Setter C, Dunst J, et al (2010). Prognostic impact of VEGF and VEGF receptor 1 (FLT1) expression in patients irradiated for stage II/III non-small cell lung cancer (NSCLC). Strahlenther Onkol, 186, 307-14.

Renyi-Vamos F, Tovari J, Fillinger J, et al (2005). Lymphangiogenesis correlates with lymph node metastasis, prognosis, and angiogenic phenotype in human non-small cell lung cancer. Clin Cancer Res, 11, 7344-53.

Saad RS, Liu Y, Han H, et al (2004). Prognostic significance of HER2/neu, p53, and vascular endothelial growth factor expression in early stage conventional adenocarcinoma and bronchioloalveolar carcinoma of the lung. Mod Pathol, 17, 1235-42.

Saintigny P, Kambouchner M, Ly M, et al (2007). Vascular endothelial growth factor-C and its receptor VEGFR-3 in non-small-cell lung cancer: concurrent expression in cancer cells from primary tumour and metastatic lymph node. Lung Cancer, 58, 205-13.

Schiller JH, Larson T, Ou SH, et al (2009). Efficacy and safety of axitinib in patients with advanced non-small-cell lung cancer: results from a phase II study. J Clin Oncol, 27, 3836-41.

Seto T, Higashiyama M, Funai H, et al (2006). Prognostic value of expression of vascular endothelial growth factor and its flt- 1 and KDR receptors in stage I non-small-cell lung cancer. Lung Cancer, 53, 91-6.
Sheng H, Aoe M, Doihara H, et al (2000). Prognostic value of vascular endothelial growth factor expression in primary lung carcinoma. Acta Med Okayama, 54, 119-26.

Shibusa T, Shijubo N, Abe S (1998). Tumor angiogenesis and vascular endothelial growth factor expression in stage I lung adenocarcinoma. Clin Cancer Res, 4, 1483-7.

Shou Y, Hirano T, Gong Y, et al (2001). Influence of angiogenetic factors and matrix metalloproteinases upon tumour progression in non-small-cell lung cancer. Br J Cancer, 85, 1706-12.

Spratlin JL, Cohen RB, Eadens M, et al (2010). Phase I pharmacologic and biologic study of ramucirumab (IMC1121B), a fully human immunoglobulin G1 monoclonal antibody targeting the vascular endothelial growth factor receptor-2. J Clin Oncol, 28, 780-7.

Starnes SL, Pathrose P, Wang J, et al (2012). Clinical and molecular predictors of recurrence in stage I non-small cell lung cancer. Ann Thorac Surg, 93, 1606-12.

Stead LF, Berri S, Wood HM, et al (2012). The transcriptional consequences of somatic amplifications, deletions, and rearrangements in a human lung squamous cell carcinoma. Neoplasia, 14, 1075-86.

Sternberg CN, Davis ID, Mardiak J, et al (2010). Pazopanib in locally advanced or metastatic renal cell carcinoma: results of a randomized phase III trial. J Clin Oncol, 28, 1061-8.

Sun JG, Wang Y, Chen ZT, et al (2009). Detection of lymphangiogenesis in non-small cell lung cancer and its prognostic value. J Exp Clin Cancer Res, 28, 21.

Takanami I, Tanaka F, Hashizume T, et al (1997). Vascular endothelial growth factor and its receptor correlate with angiogenesis and survival in pulmonary adenocarcinoma. Anticancer Res, 17, 2811-4.

Tanaka F, Ishikawa S, Yanagihara K, et al (2002). Expression of angiopoietins and its clinical significance in non-small cell lung cancer. Cancer Res, 62, 7124-9.

Tanaka F, Yanagihara K, Otake Y, et al (2004). Angiogenesis and the efficacy of postoperative administration of UFT in pathologic stage I non-small cell lung cancer. Cancer Sci, 95, 371-6.

Tierney JF, Stewart LA, Ghersi D, et al (2007). Practical methods for incorporating summary time-to-event data into metaanalysis. Trials, $\mathbf{8}, 16$.

Tomita M, Matsuzaki Y, Shimizu T, et al (2005). Vascular endothelial growth factor expression in $\mathrm{pN} 2$ non-small cell lung cancer: lack of prognostic value. Respirol, 10, 31-5.

Ucvet A, Kul C, Gursoy S, et al (2011). Prognostic value of epithelial growth factor receptor, vascular endothelial growth factor, E-cadherin, and p120 catenin in resected non-small cell lung carcinoma. Arch Bronconeumol, 47, 397-402.

Vermeulen PB, Gasparini G, Fox SB, et al (2002). Second international consensus on the methodology and criteria of evaluation of angiogenesis quantification in solid human tumours. Eur J Cancer, 38, 1564-79.

Volm M, Koomagi R, Mattern J (1997). Prognostic value of vascular endothelial growth factor and its receptor Flt-1 in squamous cell lung cancer. Int J Cancer, 74, 64-8.

Walter SD (1997). Variation in baseline risk as an explanation of heterogeneity in meta-analysis. Stat Med, 16, 2883-900.

Wang M, Liu BG, Yang ZY, et al (2012). Significance of survivin expression: Prognostic value and survival in stage III nonsmall cell lung cancer. Exp Ther Med, 3, 983-8.

Yamashita T, Uramoto H, Onitsuka T, et al (2010). Association between lymphangiogenesis-/micrometastasis- and adhesion-related molecules in resected stage I NSCLC. Lung Cancer, 70, 320-8.

Yan W, Wistuba, II, Emmert-Buck MR, et al (2011). Squamous cell carcinoma-similarities and differences among anatomical 
sites. Am J Cancer Res, 1, 275-300.

Yano T, Tanikawa S, Fujie T, et al (2000). Vascular endothelial growth factor expression and neovascularisation in nonsmall cell lung cancer. Eur J Cancer, 36, 601-9.

Yi J, Pan T (2004). Expression of vascular endothelial growth factor $\mathrm{C}$ and its receptor VEGF-R3 and their significance in non-small cell lung cancer. Zhongguo Fei Ai Za Zhi, 7, 488-92.

Yilmaz A, Ernam D, Unsal E, et al (2007). Vascular endothelial growth factor immunostaining correlates with postoperative relapse and survival in non-small cell lung cancer. Arch Med Res, 38, 764-8.

Yoo J, Jung JH, Lee MA, et al (2007). Immunohistochemical analysis of non-small cell lung cancer: correlation with clinical parameters and prognosis. J Korean Med Sci, 22, 318-25.

Yurdakul A, Akyurek N, Yilmaz S, et al (2012). Prognostic impact of matrix metalloproteinases (MMP-9 and MMP-2) and vascular endothelial growthfactor expression in nonsmall cell lung cancer. Turkish J Med Sci, 42, 281-8.

Zhan P, Wang J, Lv XJ, et al (2009). Prognostic value of vascular endothelial growth factor expression in patients with lung cancer: a systematic review with meta-analysis. $J$ Thorac Oncol, 4, 1094-103.

Zhang HZ, Wei YP, Li HG, et al (2006). Expressions of vascular endothelial growth factor $\mathrm{C}$ and matrix metalloproteinases-2 and prognosis of non-small cell lung carcinoma. Nan Fang Yi Ke Da Хие Хие Bao, 26, 1307-10.

Zhang L, Meng L, Wang L, et al (2004). The clinical significance of detection of vascular endothelial growth factor and CD44v6 expression in human non-small cell lung cancer. Zhongguo Fei Ai Za Zhi, 7, 427-30.

Zhou S, Xu S, Zhang H, et al (2007). Prognostic significance of angiogenesis and blood vessel invasion in stage I non-small cell lung cancer after complete surgical resection. Zhongguo Fei Ai Za Zhi, 10, 29-33. 